\title{
One-Step Synthesis of PEG-Coated Gold Nanoparticles by Rapid Microwave Heating
}

\author{
Seung Kwon Seol, ${ }^{1,2}$ Daeho Kim,, ${ }^{1,2}$ Sunshin Jung, ${ }^{1,2}$ Won Suk Chang, ${ }^{1}$ and Ji Tae Kim ${ }^{3}$ \\ ${ }^{1}$ Nano Hybrid Technology Research Center, Creative and Fundamental Research Division, \\ Korea Electrotechnology Research Institute (KERI), Changwon-si 642-120, Republic of Korea \\ ${ }^{2}$ University of Science \& Technology (UST), Changwon-si 642-120, Republic of Korea \\ ${ }^{3}$ Max Planck Institute for the Science of Light, 91058 Erlangen, Germany \\ Correspondence should be addressed to Seung Kwon Seol; skseol@keri.re.kr
}

Received 15 January 2013; Revised 21 February 2013; Accepted 22 February 2013

Academic Editor: Renyun Zhang

Copyright (C) 2013 Seung Kwon Seol et al. This is an open access article distributed under the Creative Commons Attribution License, which permits unrestricted use, distribution, and reproduction in any medium, provided the original work is properly cited.

Polyethylene Glycol- (PEG-) coated gold nanoparticles (PEG-AuNPs) are synthesized by a one-step route with rapid microwave heating. Homogeneous nucleation of the primary gold particles is enhanced by increasing the applied microwave power during the initial stage of the synthesis, increasing the temperature ramping rate $\left(R_{r}\right)$ and resulting in decreased size and improved uniformity of the synthesized PEG-AuNPs. Using rapid microwave heating, we successfully produce uniform colloidal PEG-AuNPs with an average diameter of $14.3 \pm 2.5 \mathrm{~nm}$ within a few minutes. By appropriate tuning of the growth parameters, microwave synthesis can produce largely colloidal PEG-AuNPs with high uniformity.

\section{Introduction}

Colloidal gold nanoparticles (AuNPs) have attracted attention due to the interesting electrical, chemical, and optical properties that have given them the potential for application in a variety of biomedical fields such as sensors [1], disease diagnosis [2-4], therapeutics $[5,6]$, and others. In particular, AuNPs can be used as image contrast and treatment agents for applications in X-ray radiography and radiotherapy for tumor treatment $[7,8]$. The goal of treatment with AuNPs in these fields is for the AuNPs to arrive at the tumor cells after administration into circulatory system and to then enhance the image contrast or treatment effect at the targeted tumor area. In general, two strategies have been developed for the targeting tumors with AuNPs: the passive route and the active targeting route $[9,10]$. The passive targeting route depends on the accumulation of AuNPs at tumor areas due to the enhanced permeation and retention (EPR) effect in tumors [11]. The active targeting route uses ligands on the AuNPs surface for recognition by binding to specific cell surface receptors on the tumor. These ligands could be small molecules, peptides, or proteins [11].
In in vivo targeting of AuNPs, it is crucial to bypass the reticuloendothelial system (RES), an essential component of the immune system composed of phagocytic cells. To evade the human body's RES, it is invariably necessary to endow AuNPs with a "stealth" function. Polyethylene Glycol (PEG) has been recommended as a capping material used to provide AuNPs with the requisite stealth against the RES [12]. Several studies have reported that PEG-coated AuNPs (PEG-AuNPs) were successful as image contrast and treatment agents for in vivo targeting $[3,13]$.

It has been recently emphasized that if PEG-AuNPs are to have practical application in the field as a biomedical agent, they need a synthesis route with mass production capability. Conventional synthesis using chemical reactants in solution is generally carried out through conductive heating with an external heat source. However, this method is inefficient for the uniform transfer of energy into the reactants, resulting in slow reaction rates and a large temperature gradient in the solution that causes nonuniform product formation.

Here, we present a rapid one-step route for preparing highly uniform, nontoxic PEG-AuNPs by microwave heating. We successfully produce uniform colloidal PEG-AuNPs with 




(a)

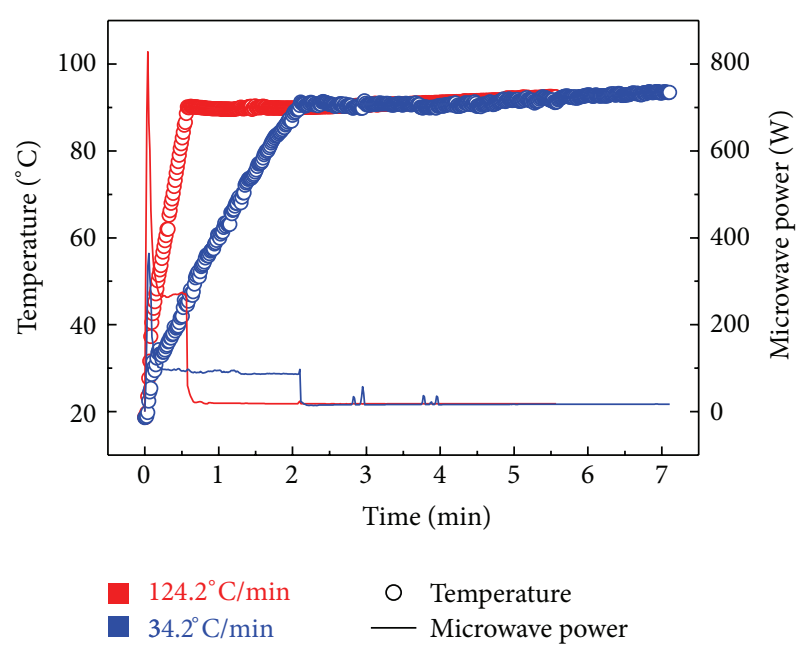

(b)

Figure 1: (a) A schematic illustration of microwave heating system. (b) Microwave power (solid line) and solution temperature (open circle) profiles during microwave synthesis of colloidal PEG-AuNPs. The two different temperature ramping rates $\left(R_{r}\right)$ of $124.2^{\circ} \mathrm{C} / \mathrm{min}$ and $34.2^{\circ} \mathrm{C} / \mathrm{min}$ are created by adjusting the applied microwave power during the initial stage (ramping region). After the temperature ramping, the solution temperature is maintained at $90^{\circ} \mathrm{C}$ using a microwave power of $10 \mathrm{~W}$ (retention region).

an average diameter of $14.3 \pm 2.5 \mathrm{~nm}$ within a few minutes of microwave heating by controlling the applied microwave power during the initial stage of synthesis.

\section{Materials and Methods}

2.1. Synthesis. A schematic illustration of the microwave heating system $(2.45 \mathrm{GHz}, 1600 \mathrm{~W})$ used for the entire synthesis of PEG-coated gold nanoparticles (PEG-AuNPs) is shown in Figure 1(a) [14-17]. In this system, the amplitude of the microwave power in the microwave cavity was calculated by measuring the difference between the forward- and backward-propagating microwaves and adjusted using a 3stub tuner module. The temperature of the reaction solution was measured using a fiber optic sensor that is not affected by microwave radiation. A feedback system between the applied microwave power and the measured temperature allowed for control of the microwave power to obtain the desired reaction temperature. The solution was placed into a $50 \mathrm{~mL}$ glass bottle and stirred with a magnetic stirrer coated with Teflon during the synthesis, and this bottle was placed in the cavity. The growth solution for one-step synthesis was prepared at room temperature by mixing $0.88 \mathrm{mM} \mathrm{HAuCl}_{4}, 5.0 \mathrm{mM}$ sodium citrate $\left(\mathrm{Na}_{3} \mathrm{Ct}\right.$ ), and $0.1 \mathrm{mM}$ PEG (average molecular weight $\sim 20,000 /$ Aldrich) in deionized water $(18.2 \mathrm{M} \Omega \cdot \mathrm{cm}) . \mathrm{Na}_{3} \mathrm{Ct}$ and PEG were used as a reducing agent and coating material, respectively.

2.2. Measurements. UV-VIS absorption spectra of the synthesized colloidal PEG-AuNPs were analyzed by a Thermo Evolution 300 spectrophotometer. TEM images were taken on a JEM-2100F (JEOL). For TEM analysis, specimens were prepared by placing a drop of colloidal PEG-AuNPs on a carbon-coated copper grid, and evaporating the solvent. The size distribution of the PEG-AuNPs was obtained by measuring the diameter of more than 200 particles. The PEGAuNPs colloidal solutions were condensed by combining centrifugation and solvent evaporation under reduced pressure. The centrifugation was performed with an Eppendorf 5810R, Germany, centrifuge and an Amicon Ultra-15, Millipore, US centrifugal filter at $4^{\circ} \mathrm{C}$. The centrifugation took place at $3200 \mathrm{~g}$ for $30 \mathrm{~min}$. The PEG-AuNPs were further condensed by a simple vacuum evaporation system. In order to reveal some of the interactions between the primary gold nanoparticles and their organic ligands, samples of PEG-AuNPs were mixed with IR grade $\mathrm{KBr}$ and subjected to FTIR analysis on a Perkin-Elmer FTIR spectrophotometer. All spectra were recorded at a resolution of $4 \mathrm{~cm}^{-1}$ over a wave number range of $600-4,000 \mathrm{~cm}^{-1}$.

2.3. Cell Viability Test. EMT-6 cells were cultured in Dulbecco's modified Eagle medium (DMEM/F-12) supplemented with $1 \%$ penicillin-streptomycin and $10 \%$ heat-inactived fetal bovine serum (Invitrogen, Carlsbad, CA) and were maintained in a humidified incubator with $5 \% \mathrm{CO}_{2}$ and at $37^{\circ} \mathrm{C}$; the culture medium was changed every two days. PEG-AuNP colloid was freshly prepared and diluted with Dulbecco's medium. After overnight cell seeding in a multiplate, the EMT- 6 cells were cocultured for 24 hours with PEG-AuNPs suspensions with different colloidal concentrations: $0.01,0.1$, or $0.5 \mathrm{mM}$. Growth medium with no nanoparticles was used for the control specimens. After incubation, some of the cells were harvested and stained by trypan blue reagent (Sigma, St. Louis, MO) to count the number of live cells.

\section{Results and Discussion}

When microwave radiation is used as a heating source for driving a chemical reaction in water or in an organic solvent with a high dipole moment, rapid volumetric heating can occur, resulting in uniform energy transfer into the solution. In addition, many nonthermal effects of microwave radiation have been previously proposed, including (i) an increase in the probability of collision between two molecules and 


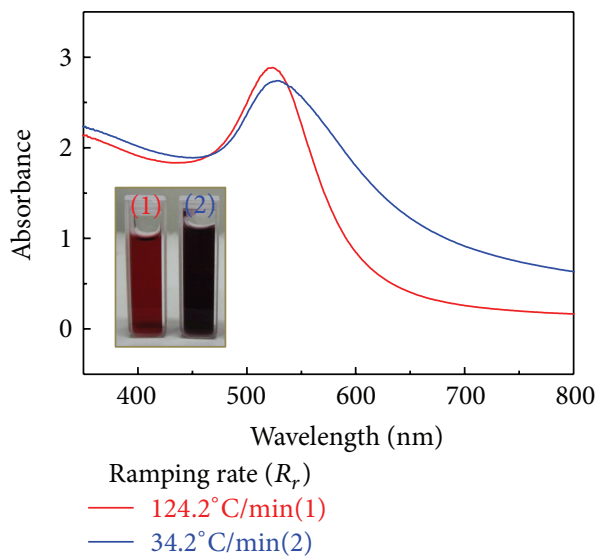

(a)

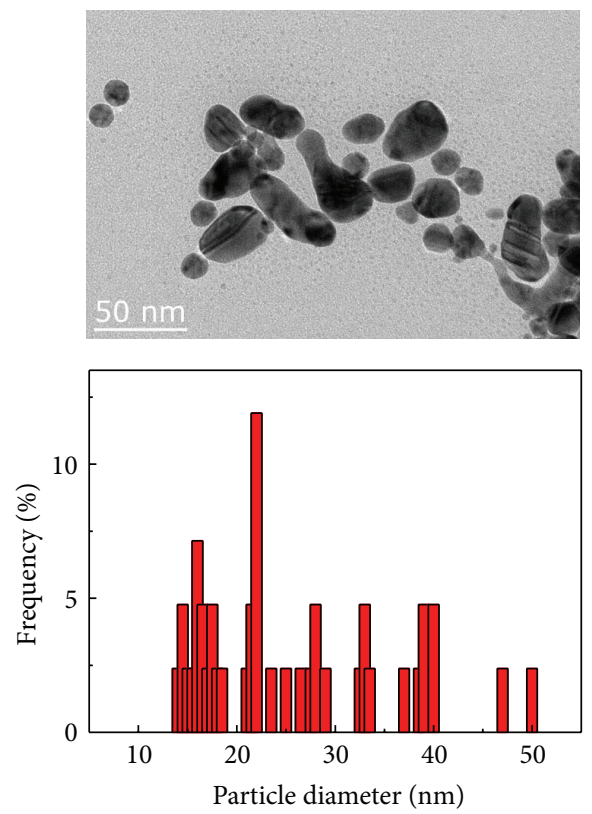

(c)


(b)

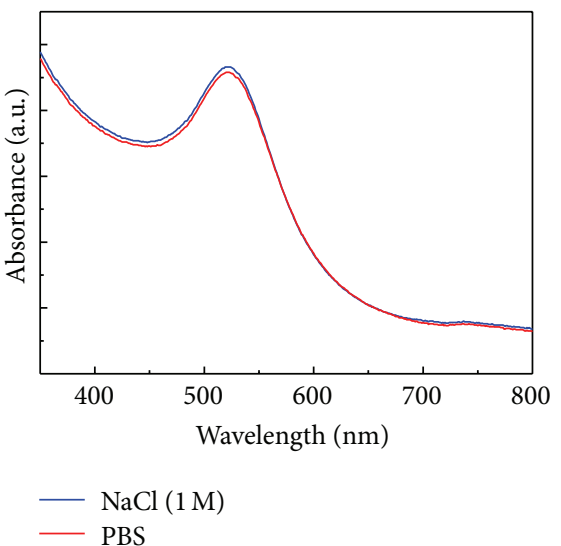

(d)

FIgURE 2: (a) UV-vis absorption spectra of the synthesized PEG-AuNPs with respect to $R_{r}$. Inset shows color of synthesized PEG-AuNPs. (b-c) TEM images and their corresponding size distributions of the synthesized PEG-AuNPs synthesized at different $R_{r}-124.2^{\circ} \mathrm{C} / \mathrm{min}(\mathrm{b})$ and $34.2^{\circ} \mathrm{C} / \mathrm{min}$ (c). (d) UV-vis spectra of PEG-AuNPs recorded at $24 \mathrm{~h}$ with PBS and $\mathrm{NaCl}$.

(ii) a decrease in the activation energy [18] resulting from the direct interaction of the electric fields with specific molecules in the reactants that could enhance reactions. These are the unique characteristics of microwave heating that improve the reaction rates, increase the yields, and increase the uniformity of the products [14-25]. We also note that microwave synthesis is one of the routes by which the need for mass production of nanoparticles can be met.
Shown in Figure 1(b) are the typical profiles of the applied microwave power (solid line) and the corresponding solution temperature (open circle) in the microwave synthesis of colloidal PEG-AuNPs. The profile can be divided into the two temperature regions of "ramping" and then "retention." In the ramping region of Figure 1(b), the solution temperature reaches the desired value of $\sim 90^{\circ} \mathrm{C}$ using two different ramping rates $\left(R_{r}\right)$ of $124.2^{\circ} \mathrm{C} / \mathrm{min}$ and $34.2^{\circ} \mathrm{C} / \mathrm{min}$. The $R_{r}$ 
is precisely controlled by adjusting the applied microwave power during the initial stage of synthesis. In the retention region, the solution temperature is maintained at $90^{\circ} \mathrm{C}$ for $5 \mathrm{~min}$ by applying a continuous microwave power of $10 \mathrm{~W}$.

Figure 2(a) shows the UV-vis absorption spectra of PEGAuNPs synthesized with the two different $R_{r}$ values and a constant retention time of $5 \mathrm{~min}$ in Figure 1(b). As the $R_{r}$ value increases from $34.2^{\circ} \mathrm{C} / \mathrm{min}$ to $124.2^{\circ} \mathrm{C} / \mathrm{min}$, the Full Width Half Maximum (FWHM) of the absorbance profile narrows and the maximum peak $\left(\lambda_{\max }\right)$ value shows a blue shift from $528 \mathrm{~nm}$ to $521 \mathrm{~nm}$, indicating the formation of colloidal PEG-AuNPs with a smaller average size and narrower size distribution. The TEM images and the corresponding size distribution of PEG-AuNPs in Figures 2(b) and 2(c) are clearly well matched with the UV-vis results of Figure 2(a). Both of these results indicate that uniform PEG-AuNPs with an average diameter of $14.3 \pm 2.5 \mathrm{~nm}$ are produced at the condition of $R_{r} \sim 124.2^{\circ} \mathrm{C} / \mathrm{min}$. Figure $2(\mathrm{~d})$ compares UV-vis absorbance spectra of PEG-AuNPs in presence of $1.0 \mathrm{M} \mathrm{NaCl}$ and 1.0 M PBS. No significant shifts in the UV-vis absorbance spectra are observed within at least $24 \mathrm{~h}$, indicating that the resulting PEG-AuNPs are stable at the conditions.

In a previous study, we investigated the formation kinetics of the microwave synthesis of sodium citrate reduced colloidal AuNPs [14]. The uniformity of the synthesized AuNPs was affected significantly by the applied microwave power during the initial stage of synthesis and by the solution $\mathrm{pH}$. The result in Figure 2 can be explained by the influence of the $R_{r}$ value on the homogeneous nucleation during the initial stage of the PEG-AuNP synthesis. The faster ramp $\left(\sim 124.2^{\circ} \mathrm{C} / \mathrm{min}\right)$ to the desired temperature of $90^{\circ} \mathrm{C}$ during the initial stage causes a higher supersaturation ratio of gold atoms in solution. This higher supersaturation results in short nucleation bursts and the production of a large number of small primary AuNPs with high uniformity. With the capping of primary AuNPs with PEG during the growth phase in the temperature retention region, the uniformity of the homogeneous primary AuNPs contributes significantly to the formation of colloidal PEG-AuNPs with a smaller average size and a narrower size distribution. The unmodified PEG molecules used in this process are unlikely to covalently bond to AuNPs; thus PEG molecules are expected to be physisorbed on the gold surfaces. The action of PEG chains is closely related to their affinity to $\mathrm{AuCl}^{-4}$ ions and conformation on the primary AuNPs $[13,15$, $26,27]$. Conversely, the extended nucleation period due to the slower $R_{r}$ of $34.2^{\circ} \mathrm{C} / \mathrm{min}$ leads to inhomogeneous nucleation of the primary AuNPs. The AuNPs nucleated during the earlier part of the temperature ramping stage are larger than those nucleated during the later part, resulting in synthesized colloidal PEG-AuNPs with a wide size distribution.

In order to confirm the PEG capping on the surface of AuNPs, we conducted the FTIR analysis of the synthesized nanoparticles. Figure 3 shows the FTIR spectra of typical of bare AuNPs, synthesized PEG-AuNPs, and pure PEG. In contrast to the spectrum of bare AuNPs, IR absorbance peaks are present in the spectrum of PEG-AuNPs, and these peaks are assigned as follows: $2,850-3,000 \mathrm{~cm}^{-1}\left(-\mathrm{CH}_{2}\right.$ stretching),



Figure 3: FTIR spectra of bare AuNPs (blue line), PEG-AuNPs (red line), and Pure PEG (black line).



FIgURE 4: Cell viability of EMT-6 cells cocultured with different concentrations of PEG-AuNPs (CM: control medium).

$1,380 \mathrm{~cm}^{-1}$ (C-H bending; $-\mathrm{CH}_{2}$ and $\left.-\mathrm{CH}_{3}\right), 1,100 \mathrm{~cm}^{-1}$ (C-O-C stretching), and $700-900 \mathrm{~cm}^{-1}$ (N-H wagging). These peaks are consistent with the spectrum of pure PEG, indicating the adsorption of PEG molecules onto the surface of AuNPs.

Biocompatibility is a critical factor to consider when evaluating the potential of nanoparticles for biomedical purposes, especially in in vivo applications such as image contrast and treatment agents. Biocompatibility is commonly verified by the assessment of the cytotoxicity of nanoparticles. The cytotoxicity of nanoparticles is estimated by measuring the overall dose-dependent toxicity of nanoparticles on cultured cells and determining cell survival after nanoparticle exposure. The cell viability results for the EMT cells exposed to different PEG-AuNPs concentrations are compared to the results for the unexposed control EMT specimen in Figure 4. The cells were treated with $0.01,0.1$, or $0.5 \mathrm{mM}$ concentrations of PEG-AuNPs and cocultured for $24 \mathrm{~h}$, ultimately exhibiting 
$>90 \%$ cell viability. These results show that the synthesized PEG-AuNPs are nontoxic particles.

\section{Conclusion}

In conclusion, we successfully synthesized highly uniform, nontoxic PEG-coated AuNPs (PEG-AuNPs) with an average diameter of $14.3 \pm 2.5 \mathrm{~nm}$ in a few minutes by a one-step route with rapid microwave heating. The formation of PEG-AuNPs is affected significantly by the applied microwave power during the initial stage of synthesis. Colloidal PEG-AuNPs with a smaller average size and a narrower size distribution are formed by homogeneous nucleation of primary AuNPs during the initial stage when using a higher temperature ramping rate $\left(R_{r}\right)$. We can anticipate that a large-volume production of PEG-AuNPs with high uniformity can be achieved using one-step microwave synthesis with appropriate control over the applied microwave power.

\section{Acknowledgments}

The authors thank Dr. C. C. Chien, Dr. C. H. Wang, and Dr. Y. Hwu (Institute of Physics, Academia Sinica, Taiwan) for their suggestions and assistance during the work. This work was supported by a Grant (code no. 13-12-N0101-06) from the Primary Research Program of Korea Electrotechnology Research Institute (KERI), Republic of Korea.

\section{References}

[1] E. Katz and I. Willner, "Integrated nanoparticle-biomolecule hybrid systems: synthesis, properties, and applications," Angewandte Chemie International Edition, vol. 43, no. 45, pp. 60426108, 2004.

[2] X. Qian, X. H. Peng, D. O. Ansari et al., "In vivo tumor targeting and spectroscopic detection with surface-enhanced Raman nanoparticle tags," Nature Biotechnology, vol. 26, no. 1, pp. 83-90, 2008.

[3] D. K. Kim, S. J. Park, J. H. Lee, Y. Y. Jeong, and S. Y. Jon, "Antibiofouling polymer-coated gold nanoparticles as a contrast agent for in vivo x-ray computed tomography imaging," Journal of the American Chemical Society, vol. 129, no. 24, pp. 7661-7665, 2007.

[4] C. C. Chien, H. H. Chen, S. F. Lai et al., "Gold nanoparticles as high resolution X-ray imaging contrast agents for the analysis of tumor-related micro-vasculature," Journal of Nanobiotechnology, vol. 10, p. 10, 2012.

[5] M. Ferrari, "cancer nanotechnology: opportunities and challenges," Nature Reviews Cancer, vol. 5, pp. 161-171, 2005.

[6] B. Kang, M. A. Mackey, and M. A. El-Sayed, "Nuclear targeting of gold nanoparticles in cancer cells induces DNA damage, causing cytokinesis arrest and apoptosis," Journal of the American Chemical Society, vol. 132, no. 5, pp. 1517-1519, 2010.

[7] J. F. Hainfeld, D. N. Slatkin, T. M. Focella, and H. M. Smilowitz, "Gold nanoparticles: a new X-ray contrast agent," British Journal of Radiology, vol. 79, no. 939, pp. 248-253, 2006.

[8] J. F. Hainfeld, D. N. Slatkin, and H. M. Smilowitz, "The use of gold nanoparticles to enhance radiotherapy in mice," Physics in Medicine and Biology, vol. 49, no. 18, pp. N309-N315, 2004.
[9] L. Brannon-Peppas and J. O. Blanchette, "Nanoparticle and targeted systems for cancer therapy," Advanced Drug Delivery Reviews, vol. 56, no. 11, pp. 1649-1659, 2004.

[10] I. Brigger, C. Dubernet, and P. Couvreur, "Nanoparticles in cancer therapy and diagnosis," Advanced Drug Delivery Reviews, vol. 54, no. 5, pp. 631-651, 2002.

[11] W. Cai, T. Gao, H. Hong, and J. Sun, "Applications of gold nanoparticles in cancer nanotechnology," Nanotechnology Science and Applications, vol. 1, pp. 17-32, 2008.

[12] T. Niidome, M. Yamagata, Y. Okamoto et al., "PEG-modified gold nanorods with a stealth character for in vivo applications," Journal of Controlled Release, vol. 114, no. 3, pp. 343-347, 2006.

[13] C. J. Liu, C. H. Wang, C. C. Chien et al., "Enhanced x-ray irradiation-induced cancer cell damage by gold nanoparticles treated by a new synthesis method of polyethylene glycol modification," Nanotechnology, vol. 19, no. 29, Article ID 295104, 2008.

[14] S. K. Seol, D. Kim, S. Jung, and Y. Hwu, "Microwave synthesis of gold nanoparticles: effect of applied microwave power and solution pH," Materials Chemistry and Physics, vol. 131, no. 1-2, pp. 331-335, 2011.

[15] S. K. Seol, D. Kim, S. Jung et al., "Effect of citrate on poly(vinyl pyrrolidone)-stabilized gold nanoparticles formed by PVP reduction in microwave (MW) synthesis," Materials Chemistry and Physics, vol. 137, no. 1, pp. 135-139, 2012.

[16] D. Kim, J. Choi, G. J. Kim et al., "Microwave-accelerated energyefficient esterification of free fatty acid with a heterogeneous catalyst," Bioresource Technology, vol. 102, no. 3, pp. 3639-3641, 2011.

[17] D. Kim, J. Choi, G. J. Kim, S. K. Seol, and S. Jung, "Accelerated esterification of free fatty acid using pulsed microwaves," Bioresource Technology, vol. 102, no. 14, pp. 7229-7231, 2011.

[18] H. M. Kingston and S. J. Haswell, Microwave-Enhanced Chemistry, American Chemical Society, Washington, DC, USA, 2005.

[19] J. A. Gerbec, D. Magana, A. Washington, and G. F. Strouse, "Microwave-enhanced reaction rates for nanoparticle synthesis," Journal of the American Chemical Society, vol. 127, no. 45, pp. 15791-15800, 2005.

[20] C. Fan, W. Li, S. Zhao, J. Chen, and X. Li, "Efficient one pot synthesis of chitosan-induced gold nanoparticles by microwave irradiation," Materials Letters, vol. 62, no. 20, pp. 3518-3520, 2008.

[21] M. Shen, Y. Du, N. Hua, and P. Yang, "Microwave irradiation synthesis and self-assembly of alkylamine-stabilized gold nanoparticles," Powder Technology, vol. 162, no. 1, pp. 64-72, 2006.

[22] F. K. Liu, Y. C. Chang, F. H. Ko, and T. C. Chu, "Microwave rapid heating for the synthesis of gold nanorods," Materials Letters, vol. 58, no. 3-4, pp. 373-377, 2004.

[23] C. Gutiérrez-Wing, J. J. . Velázquez-Salazar, and M. JoséYacamán, "Procedures for the synthesis and capping of metal nanoparticles," Methods in Molecular Biology, vol. 906, pp. 3-19, 2012.

[24] C. Gutiérrez-Wing, R. Esparza, C. Vargas-Hernández, M. E. Fernández García, and M. José-Yacamán, "Microwaveassisted synthesis of gold nanoparticles self-assembled into selfsupported superstructures," Nanoscale, vol. 4, pp. 2281-2287, 2012.

[25] M. N. Nadagouda, T. F. Speth, and R. S. Varma, "Microwaveassisted green synthesis of silver nanostructures," Accounts of Chemical Research, vol. 19, no. 7, pp. 469-478, 2011. 
[26] C. H. Wang, C. J. Liu, C. L. Wang et al., "Optimizing the size and surface properties of polyethylene glycol (PEG)-gold nanoparticles by intense x-ray irradiation," Journal of Physics D, vol. 41, no. 19, Article ID 195301, 2008.

[27] C. E. Hoppe, M. Lazzari, I. Pardiñas-Blanco, and M. A. LópezQuintela, "One-step synthesis of gold and silver hydrosols using poly(N-vinyl-2- pyrrolidone) as a reducing agent," Langmuir, vol. 22, no. 16, pp. 7027-7034, 2006. 



Submit your manuscripts at http://www.hindawi.com
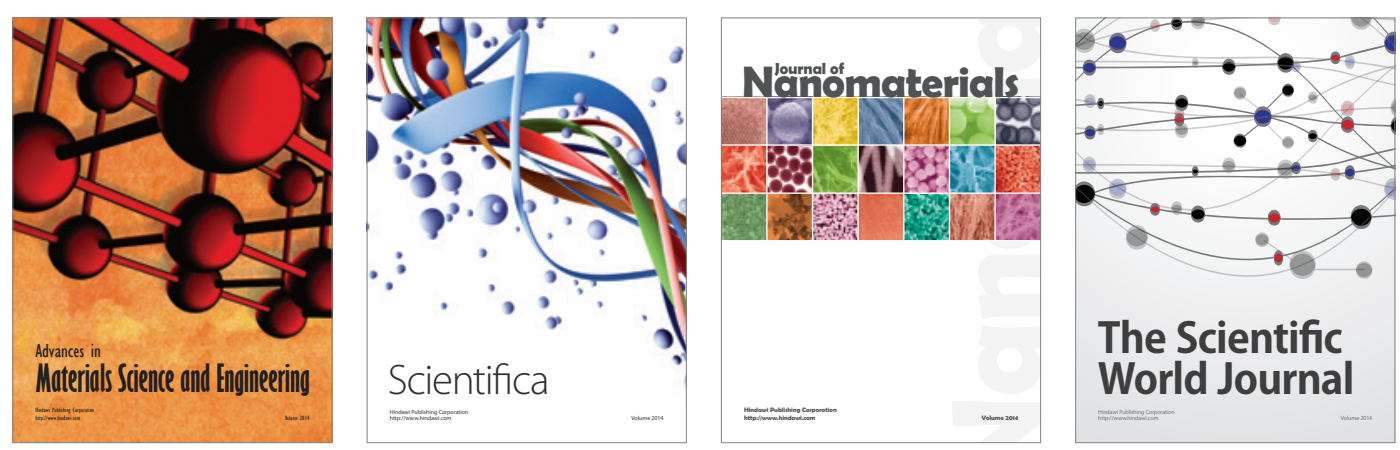

\section{The Scientific World Journal}
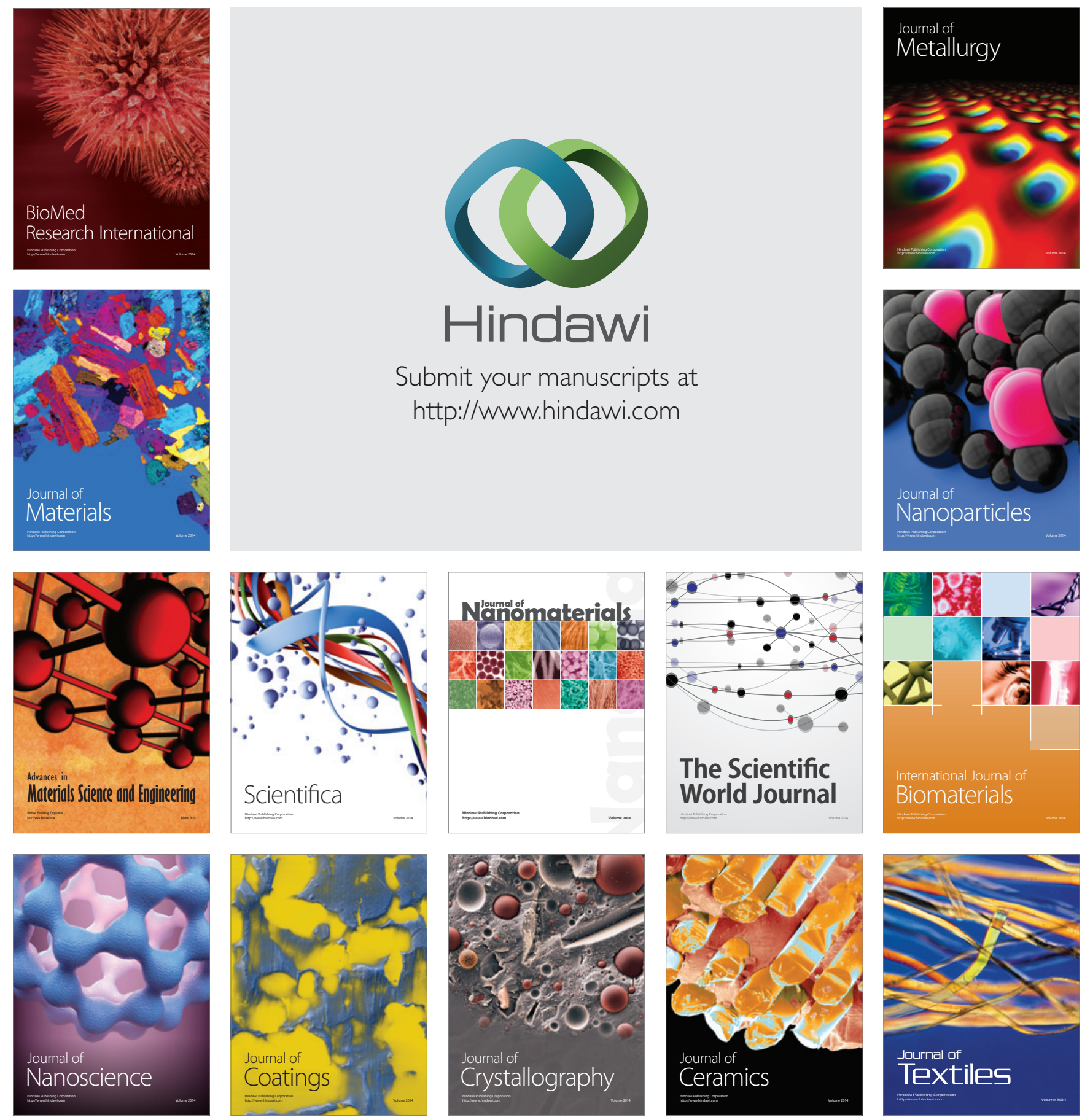